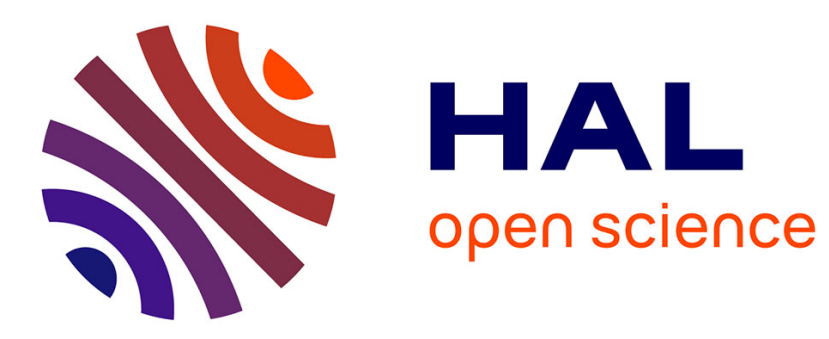

\title{
Nonlinear Hamiltonian modelling of magnetic shape memory alloy based actuators.
}

Jean-Yves Gauthier, Arnaud Hubert, Joël Abadie, Nicolas Chaillet, Christian Lexcellent

\section{- To cite this version:}

Jean-Yves Gauthier, Arnaud Hubert, Joël Abadie, Nicolas Chaillet, Christian Lexcellent. Nonlinear Hamiltonian modelling of magnetic shape memory alloy based actuators. Sensors and Actuators A: Physical , 2008, 141, pp.536-547. 10.1016/j.sna.2007.10.012 . hal-00206175

\section{HAL Id: hal-00206175 https://hal.science/hal-00206175}

Submitted on 16 Jan 2008

HAL is a multi-disciplinary open access archive for the deposit and dissemination of scientific research documents, whether they are published or not. The documents may come from teaching and research institutions in France or abroad, or from public or private research centers.
L'archive ouverte pluridisciplinaire HAL, est destinée au dépôt et à la diffusion de documents scientifiques de niveau recherche, publiés ou non, émanant des établissements d'enseignement et de recherche français ou étrangers, des laboratoires publics ou privés. 


\title{
Nonlinear Hamiltonian modelling of Magnetic Shape Memory Alloy based actuators
}

\author{
Jean-Yves Gauthier $^{*, a}$, Arnaud Hubert ${ }^{\mathrm{a}}$, Joël Abadie ${ }^{\mathrm{a}}$, Nicolas Chaillet ${ }^{\mathrm{a}}$, Christian Lexcellent $^{\mathrm{b}}$ \\ ${ }^{a}$ Laboratoire d'Automatique de Besançon, UMR CNRS 6596, ENSMM, UFC, 24 rue Alain Savary, 25000 Besançon, France \\ ${ }^{\mathrm{b}}$ Institut Femto-st, UMR CNRS 6174, ENSMM, UFC, UTBM, 24 chemin de l'Epitaphe, 25000 Besançon, France
}

\begin{abstract}
This paper proposes an application of the Lagrangian formalism and its Hamiltonian extension to design, model and control a mechatronic system using Magnetic Shape Memory Alloys. In this aim, an original dynamical modelling of a Magnetic Shape Memory Alloy based actuator is presented. Energy-based techniques are used to obtain a coherent modelling of the magnetical, mechanical and thermodynamic phenomena. The Lagrangian formalism, well suited in such a case, is introduced and used to take into account the dynamical effects. Hamilton equations are deduced and used for the computation of the theoretical behaviour of this actuator. These numerical simulations are compared with some experimental measurements permitting the validation of the proposed modelling. Beyond the work presented here, these results will be used to design an energy shaping nonlinear control well-adapted for a strongly nonlinear active material.
\end{abstract}

Key words: Micro-Mechatronic, Nonlinear Dynamics, Active Materials, Magnetic Shape Memory Alloys, Hamiltonian and Lagrangian Modelling

\section{Introduction}

In engineering fields, the current trend is to design smaller and smaller components. The field of mechatronics is not an exception and leads to the emergence of the micro-mechatronic field. In this case sensors and actuators design is more and more dominated by the use of active materials. Most of these materials permit simultaneously actuation and sensing functions in an integrated and distributed way. This integration makes the implementation easier at the mini- and microscopic scale as compared with the classical design of the multi-components mechatronics systems.

Among the current offer of active materials (see [1] for a recent review), Magnetic Shape Memory Alloys (MSMAs) are interesting candidates because their performances are situated between piezoelectric materials (high frequencies but small strain) and classical Shape Memory Alloys (SMAs) (large strain but low frequencies). As shown in numerous works performed by the material research community, the strongly nonlinearity of the MSMAs behaviour

\footnotetext{
* Corresponding author.

Email address: jygauthi@ens2m.fr (Jean-Yves Gauthier).
}

will be undoubtedly an issue for the design and the control of innovative systems based on MSMAs. In previous works the authors of the present paper have proposed a quasi-static modelling for MSMAs with a reasonable prediction [2]. This model is based on the thermodynamics of irreversible processes because it can model hysteretic and nonlinear material behaviours. In our case, this model gives the mechanical strain of the MSMA as a function of the mechanical stress and the magnetic field. Nevertheless, in order to design new actuators and their efficient control laws, this model has to be extented to the dynamic case. In this way, a relevant approach lies in the use of an energetic and variational point of view. In this paper, we propose to model a complete MSMA actuator by using the Lagrangian formalism. This formalism permits a relevant and coherent approach to model a complete mechatronic system. Beyond the work presented here, this modelling will permit in future works to explore some recent nonlinear control techniques based on energy functions such as Lyapunov based design [3], passivity based control [4], energy shaping and damping assignment [5] and Port Hamiltonian Control [6].

A description of MSMA properties will be first reported. Then the lagrangian and hamiltonian formalisms will be 
shortly described. Then, the modelling of an MSMA based actuator including its mechanical load is proposed. Simulations and experimental results are finally compared with some conclusions and perspectives.

\section{Magnetic Shape Memory Alloys (MSMA)}

\subsection{MSMA properties and characteristics}

The value of the maximum reachable strain is an important property to design actuators using active materials. Classical SMA are one of the active materials that provide the largest strains. Nevertheless, they present a low response time because of the energy conversion involving a heat transfer. A new SMA sensitive to the magnetic field was obtained in 1995/1996 by some research teams in the USA, at the MIT for the Ni-Mn-Ga alloy [7] and at the University of Minnesota for the FePd alloy [8]. Since then these materials knew some improvements concerning mainly the working temperature range and the maximum available strain. When actuating by magnetic fields, these materials permit now to obtain a large strain (6 to $10 \%$ ) with a response time shorter than the classical SMA. Today this time is in the range of the millisecond. The MSMA used in this paper is a non-stoechiometric Ni-Mn-Ga monocrystal corresponding to the most currently used MSMA material.

In this alloy, the martensite phase can appear in three different variants corresponding to the three possible crystallographic directions in the sample (see Fig. 1 (a)).

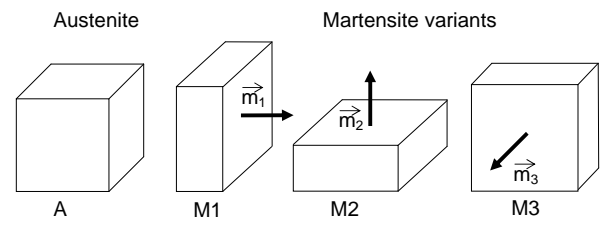

(a)

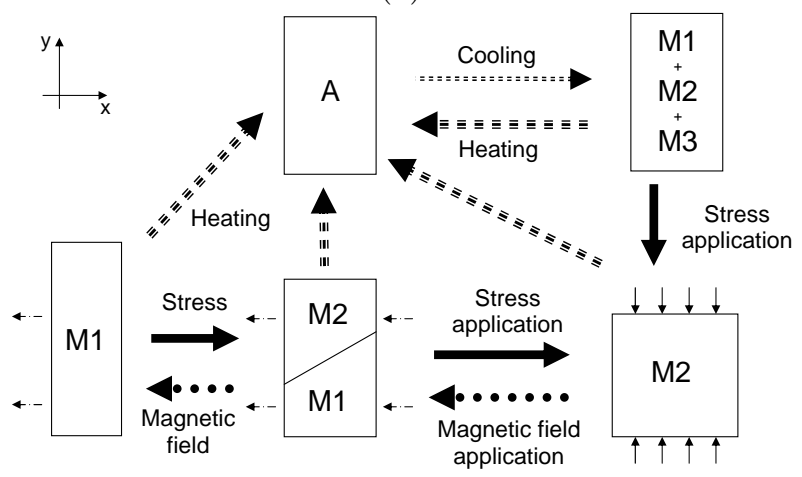

(b)

Fig. 1. MSMA behaviour: (a) austenite phase and the three martensite variants, (b) martensitic reorientation: effects of mechanical stress, magnetic field and temperature.

The martensitic reorientation principle is presented in Fig. 1 (b): at high temperature, the MSMA sample is in austenitic phase (A). After a cooling process, the austenite phase is transformed into a martensite phase without any favoured variants. The resulting sample contains therefore martensite variants into three equal portions (M1, M2 and M3). If a mechanical stress is applied in a specific direction, then the fraction of variant with its short axis in this direction grows. If this stress is high enough then the sample will only contain this variant (for example M1 in Fig. 1 (b)). If the stress decreases, the volume fraction of the M1 variant does not completely decrease due in part to a large hysteresis. In the same way, if a magnetic field is applied, the variant which has its easy magnetization direction in the field direction is favoured. As the easy magnetization direction is the same as the short axis of the M2 variant, this variant fraction increases as shown in Fig. 1 (b) by the application of a magnetic field perpendicular to the stress field. The distribution between the magnetic field and the mechanical stress permits to control the macroscopic strain. With a pre-stress, one can then obtain an actuator driven by the magnetic field only. By heating, austenite phase can also be recovered.

Beyond these interesting properties, it must be noticed that this material has also some important drawbacks like the brittleness of the single-crystal, the high required magnetic field $(400 \mathrm{kA} / \mathrm{m})$, the large dependence of the material parameters on temperature changes, a small blocking stress (2-3 MPa) and a large hysteretical thermo-magnetomechanical behaviour.

\subsection{MSMA quasi-static modelling}

\subsubsection{Introduction}

The quasi-static modelling of the MSMA is based on the thermodynamics of irreversible processes with internal variables because this subject can take into account the strong nonlinear behaviour of this kind of materials. The resulting model permits to express the behaviour of the material including all the thermo-magneto-mechanical couplings. More details concerning the modelling approach can be found in [2] and only the key points of this modelling are reported in this paper.

As the active material is used in this paper in a two dimensional actuation way (in the xy plane shown in Fig. 1), the MSMA sample can be considered as constituted by only two martensite variants M1 and M2. The internal variable $z$ and its complement $(1-z)$ are respectively the M1 and the M2 volume fractions. A magnetic field $H$ and a mechanical stress $\sigma$ are applied to the MSMA sample as described in the section 2.1 and in Fig. 1 (b). The total strain $\varepsilon$ of the MSMA sample can be separated into an elastic strain $\frac{\sigma}{E}$ and reorientation strain of the martensite variants $\gamma \cdot z . E$ is the Young modulus of the MSMA, and $\gamma$ is the maximum strain due to the reorientation process of martensite variants. In addition, an interaction between these two variants takes place. The energy corresponding to this interaction depends on martensite fractions and on 
a specific parameter $K_{12}$.

\subsubsection{Helmholtz free energy}

An Helmholtz free energy $\mathcal{F}$ of the MSMA sample was proposed in [2]. It can be divided into four parts: $\mathcal{F}=$ $\mathcal{F}_{\text {chem }}+\mathcal{F}_{\text {therm }}+\mathcal{F}_{\text {mech }}+\mathcal{F}_{\text {mag }}$. In this paper, because we are mainly focused on the reorientation process $(\mathrm{M} 1 \rightleftharpoons \mathrm{M} 2)$ and not on the transformation process $(\mathrm{A} \rightleftharpoons \mathrm{M})$, the chemical energy $\mathcal{F}_{\text {chem }}$ linked to a phase transition does not change and can be neglected for the variational computations. Moreover there is also no variation of the thermal energy $\mathcal{F}_{\text {therm }}$ because the temperature is kept constant in the actuator principle described in this paper.

The Helmholtz energy will then not take into account these two contributions. $\mathcal{F}_{\text {mech }}$ takes into account the elastic energy of the MSMA, the energy due to the reorientation of M1 and M2 martensite variants and the interaction energy $\mathcal{F}_{\text {int }}=K_{12} \cdot z \cdot(1-z)$ between these two variants [9]:

$$
\mathcal{F}_{\text {mech }}(\varepsilon, z)=\frac{E}{2}(\varepsilon-\gamma z)^{2}+K_{12} \cdot z \cdot(1-z)
$$

$\mathcal{F}_{\text {mag }}$ is the MSMA magnetic energy.

$$
\mathcal{F}_{\text {mag }}(B, z)=\int_{0}^{B} H(b) d b
$$

The latter is detailed in the following section.

\subsubsection{MSMA magnetic behaviour}

For the MSMA magnetic modelling using the thermodynamics of irreversible processes with internal variables, two internal variables are considered. Fig. 2 shows the representative elementary volume as proposed in [10]. $\alpha(H)$ is the Weiss domain width and $\theta(H)$ is the magnetization rotation angle. If no magnetic field is applied, $\alpha$ and $\theta$ are equal to zero. When a small magnetic field is applied in the $x$ direction, $\alpha$ increases corresponding to a magnetization in the M1 easy magnetization direction. When a larger magnetic field is applied, $\theta$ increases to obtain a magnetization in the same direction as the direction of the applied magnetic field. This process corresponds to a magnetization in the M2 hard magnetization direction. From Fig. 2, the magnetization in the $x$ direction can be expressed as:

$$
M(z, H)=M_{S}[(2 \alpha(H)-1) z+\sin (\theta(H))(1-z)]
$$

We choose to use a linear with saturation behaviour of $M(H)$ when $z$ is kept constant. Then, in this case, the following functions for $\alpha(H)$ and $\theta(H)$ can be used:

$$
\begin{gathered}
0 \leqslant \alpha=\frac{\chi_{a} H}{2 M_{S}}+\frac{1}{2} \leqslant 1 \\
-\frac{\pi}{2} \leqslant \theta=\arcsin \left(\frac{\chi_{t} H}{M_{S}}\right) \leqslant \frac{\pi}{2}
\end{gathered}
$$

$\chi_{a}$ and $\chi_{t}$ are respectively the magnetic susceptibilities in the easy and hard magnetization directions of the martensite. The anisotropic energy is taken into account by the way of these two parameters. Moreover, the saturation behaviour is rendered by the use of the magnetisation saturation level $M_{S}$.

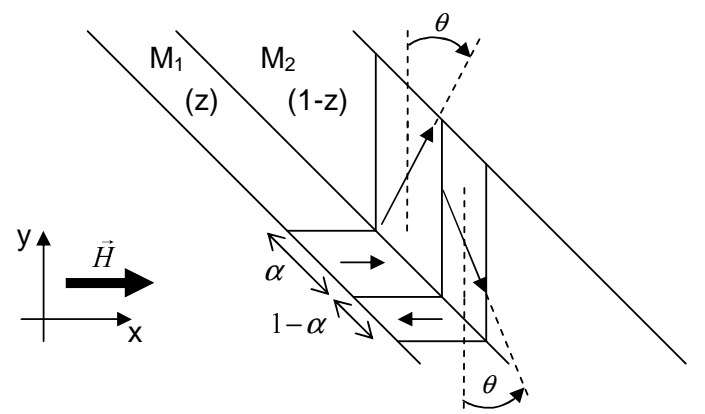

Fig. 2. Representative elementary volume, arrows represent local domains magnetizations [10].

\subsubsection{Clausius-Duhem inequality}

As seen in the scientific literature concerning MSMA, the mechanical behaviour of this material is highly irreversible. In the thermodynamics of irreversible processes framework, the irreversibility is described and expressed by the Clausius-Duhem inequality. In [2], we propose for the MSMAs, the following inequality:

$$
d \mathcal{D}=-\rho \cdot d \mathcal{F}(\sigma, H, z)+H d B+\sigma d \varepsilon \geqslant 0
$$

This expression can be reduced to:

$$
d \mathcal{D}=\pi^{f *} d z \geqslant 0
$$

$\pi^{f *}$ is the thermodynamic force associated with $z$ and is defined as:

$$
\pi^{f *}=-\frac{\partial \mathcal{F}}{\partial z}=\sigma \gamma-K_{12}(1-2 z)+\pi_{m a g}^{f}(\alpha, \theta)
$$

This equation express the distribution between the mechanical force associated with the mechanical compressive stress $\sigma<0$ and the mechanical force associated with the magnetic field $\pi_{\text {mag }}^{f}(\alpha, \theta)$. The expression of $\pi_{\text {mag }}^{f}(\alpha, \theta)$ is more easily computed using a Legendre transformation between $B$ and $H$ :

$$
\begin{aligned}
\pi_{\text {mag }}^{f}(\alpha, \theta) & =-\frac{\partial}{\partial z}\left(\int_{0}^{B} H(b) d b\right) \\
& =\frac{\partial}{\partial z}\left(\int_{0}^{H} B(h, z) d h\right) \\
& \left.=\frac{\partial}{\partial z}\left(\int_{0}^{H} \mu_{o}(M(h, z)+h)\right) d h\right) \\
& =\frac{\partial}{\partial z}\left(\int_{0}^{H} \mu_{o} M(h, z) d h\right) \\
& =\mu_{o} M_{S}^{2}\left[\frac{(1-2 \alpha) \sin \theta}{\chi_{t}}+\frac{(2 \alpha-1)^{2}}{2 \chi_{a}}+\frac{\sin ^{2} \theta}{2 \chi_{t}}\right]
\end{aligned}
$$

In addition, in order to satisfy the Clausius-Duhem inequality, one have to ensure that:

$$
\begin{aligned}
& \text { if } \pi^{f *} \geq 0, \dot{z} \geq 0 \\
& \text { if } \pi^{f *} \leq 0, \dot{z} \leq 0
\end{aligned}
$$

It indeed permits to represent some hysteretical processes. For example, in a major loop, i.e. a complete rearrangement 
from $z=0$ to $z=1$ (path a) and from $z=1$ to $z=0$ (path b) as reported in Fig. 3, the rearrangement begins when $\pi^{f *} \geqslant \pi^{c r}$ for the path a and when $\pi^{f *} \leqslant-\pi^{c r}$ for the path $b$. After the rearrangement starts, the behaviour can be represented by the following kinetic equation:

$$
\dot{\pi}^{f *}=\lambda_{C} \dot{z}
$$

In this case, it corresponds to a linear piecewise discretization.

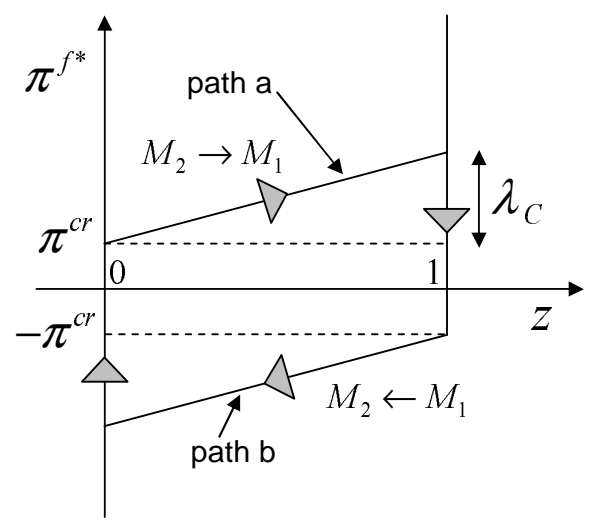

Fig. 3. The thermodynamic force $\pi^{f *}$ according to the internal variable $z$ for an hysteretical behaviour.

\section{Lagrangian and Hamiltonian formalisms}

\subsection{The Lagrangian formalism}

The lagrangian formalism is a modelling technique based on some energy functions used in addition with the Hamilton principle [11] [12]. This principle postulates that the variation of an action $\mathcal{S}$ between two times on a real path is always equal to zero. This action is the lagrangian $\mathcal{L}(\mathbf{q}, \dot{\mathbf{q}}, t)$ integrated between the two times $t_{1}$ et $t_{2}$ (see Fig. 4 ).

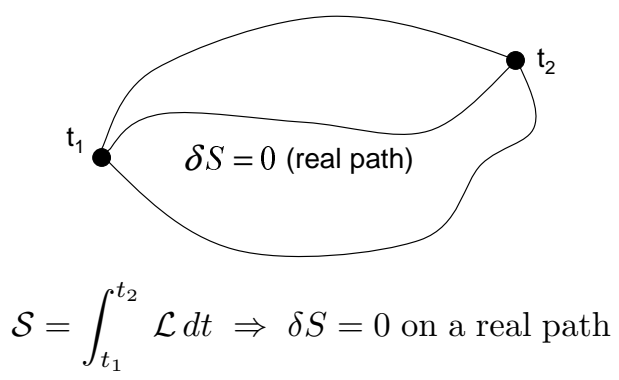

Fig. 4. Variation of the action in the Hamilton principle.

In the case of non relativist systems, the lagrangian function is the difference between a kinetic co-energy $\mathcal{T}^{*}(\dot{\mathbf{q}})$ and a potential energy $\mathcal{V}(\mathbf{q})[13]$.

$$
\mathcal{L}(\mathbf{q}, \dot{\mathbf{q}})=\mathcal{T}^{*}(\dot{\mathbf{q}})-\mathcal{V}(\mathbf{q})
$$

In the case of conservative systems (i.e. closed and nondissipative: $\mathcal{L}=\mathcal{L}(\mathbf{q}, \dot{\mathbf{q}}))$, a variational calculus on the Hamilton principle leads to the set of $n$ Lagrange equations:

$$
\frac{\partial \mathcal{L}}{\partial q_{i}}-\frac{d}{d t}\left(\frac{\partial \mathcal{L}}{\partial \dot{q}_{i}}\right)=0 \quad i \in[1, n]
$$

$\frac{\partial \mathcal{L}}{\partial q_{i}}$ are generalized forces and $p_{i}=\frac{\partial \mathcal{L}}{\partial \dot{q}_{i}}$ are generalized momentums. This formalism can be extended to the nonconservative case (open and dissipative systems) including kinematic constraints using an extented lagrangian function $\mathcal{L}^{\prime}$ and the Lagrange multipliers technique:

- the external generalized forces $\mathbf{f}_{\text {ext }}(\mathbf{q}, t)$ are taking into account in the variation of $\mathcal{L}^{\prime}$ by adding their virtual works $\delta \mathcal{W}_{\text {ext }}=\mathbf{f}_{\text {ext }}(\mathbf{q}, t) \cdot \delta \mathbf{q}$;

- dissipations by static and viscous frictions are taking into account by adding their dissipated energies variations $\delta \mathcal{Q}_{s}(\mathbf{q})$ and $\delta \mathcal{Q}_{v}(\dot{\mathbf{q}})$. The dissipation by viscous friction $\mathcal{Q}_{v}(\dot{\mathbf{q}})$ is calculated with a Rayleigh dissipation function $\mathcal{R}(\dot{\mathbf{q}}): \mathcal{Q}_{v}(\dot{\mathbf{q}})=\int_{t_{1}}^{t_{2}} \mathcal{R}(\dot{\mathbf{q}}) d t ;$

- the holonomic kinematic constraints $\mathbf{c}(\mathbf{q})=0$ are taking into account with a Lagrange multipliers technique by adding the term $-\lambda \cdot \delta \mathbf{c}(\mathbf{q})$ to the variation $\delta \mathcal{L}^{\prime}$.

Finally, we have:

$$
\delta \mathcal{L}^{\prime}=\delta \mathcal{L}+\mathbf{f}_{\text {ext }} \cdot \delta \mathbf{q}+\delta \mathcal{Q}_{s}+\delta \mathcal{Q}_{v}-\lambda \cdot \delta \mathbf{c}
$$

The Hamilton principle using $\delta \mathcal{L}^{\prime}$ gives the following Lagrange equations:

$$
\frac{\partial \mathcal{L}}{\partial q_{i}}-\frac{d}{d t}\left(\frac{\partial \mathcal{L}}{\partial \dot{q}_{i}}\right)-\frac{\partial \mathcal{R}}{\partial \dot{q}_{i}}+\frac{\partial \mathcal{Q}_{s}}{\partial q_{i}}+f_{\text {ext }, i}-\lambda_{i} \cdot \frac{\partial c_{i}}{\partial q_{i}}=0
$$

More details concerning these modelling techniques can be found in [13] for electrical and electromechanical systems and in [14] and [15] for mechatronic and distributed systems.

\subsection{The Hamilton formalism}

The Hamilton formalism is an extension of the lagrangian formalism which uses a Legendre transformation to substitute the time rate functions $\dot{\mathbf{q}}$ in the lagrangian $\mathcal{L}(\mathbf{q}, \dot{\mathbf{q}})$ for the generalized momentum $\mathbf{p}=\frac{\partial \mathcal{L}}{\partial \dot{\mathbf{q}}}$ in a new energy function called the hamiltonian function $\mathcal{H}(\mathbf{q}, \mathbf{p})$ :

$$
\mathcal{H}(\mathbf{q}, \mathbf{p})=\mathbf{p} \cdot \dot{\mathbf{q}}-\mathcal{L}(\mathbf{q}, \dot{\mathbf{q}})
$$

In the case of non relativist systems, the hamiltonian function corresponds to the total energy expressed with coordinates $\mathbf{q}$ and momentums $\mathbf{p}$ instead of coordinates $\mathbf{q}$ and velocities $\dot{\mathbf{q}}$ :

$$
\mathcal{H}(\mathbf{q}, \mathbf{p})=\mathcal{T}(\mathbf{p})+\mathcal{V}(\mathbf{q})
$$

Then the $n$ second order Lagrange equations are transformed into a set of $2 n$ first order Hamilton equations:

$$
\left\{\begin{array}{l}
\dot{q}_{i}=\frac{\partial \mathcal{H}}{\partial p_{i}} \\
\dot{p}_{i}=-\frac{\partial \mathcal{H}}{\partial q_{i}}
\end{array} \quad i \in[1, n]\right.
$$

The extented lagrangian function for a controlled dissipative system with kinematic constraints can also be transformed into an extented hamiltonian function. This leads to the following Hamilton equations: 


$$
\left\{\begin{array}{l}
\dot{q}_{i}=\frac{\partial \mathcal{H}}{\partial p_{i}} \\
\dot{p}_{i}=-\frac{\partial \mathcal{H}}{\partial q_{i}}-\frac{\partial \mathcal{R}}{\partial \dot{q}_{i}}+\frac{\partial \mathcal{Q}_{s}}{\partial q_{i}}+f_{\text {ext }, i}-\lambda_{i} \cdot \frac{\partial c_{i}}{\partial q_{i}}
\end{array}\right.
$$

The recent control techniques called Port-Hamiltonian use this set of equations in a matrix form. In addition, it uses a specific writing to characterize the connections between different coordinates. For a conservative system, this gives $[5]$ :

$$
\frac{d}{d t}\left(\begin{array}{c}
q_{i} \\
p_{i}
\end{array}\right)=\underbrace{\left(\begin{array}{cc}
\mathbf{0} & \mathbf{I} \\
-\mathbf{I} & \mathbf{0}
\end{array}\right)}_{\mathbf{J}} \cdot\left(\begin{array}{c}
\frac{\partial \mathcal{H}}{\partial q_{i}} \\
\frac{\partial \mathcal{H}}{\partial p_{i}}
\end{array}\right)
$$

The extension to a controlled dissipative system with kinematic constraints is also possible by using a matrix A representing the kinematic constraints between the different coordinates $q_{i}$, a dissipation matrix $\mathbf{R}$ including the viscous and static frictions and finally a matrix $\mathbf{B}$ and a control input $\mathbf{u}(t)$ to take into account the external forces:

$\frac{d}{d t}\left(\begin{array}{l}\mathbf{q} \\ \mathbf{p}\end{array}\right)=\left(\begin{array}{cc}\mathbf{0} & \mathbf{I} \\ -\mathbf{I} & -\mathbf{R}\end{array}\right) \cdot\left(\begin{array}{l}\frac{\partial \mathcal{H}}{\partial \mathbf{q}} \\ \frac{\partial \mathcal{H}}{\partial \mathbf{p}}\end{array}\right)+\left(\begin{array}{l}\mathbf{0} \\ \mathbf{A}\end{array}\right) \cdot \lambda+\left(\begin{array}{l}\mathbf{0} \\ \mathbf{B}\end{array}\right) \cdot \mathbf{u}(t)$

\section{Description of the MSMA actuator}

The mechatronic system considered in this paper is a simple actuator including an MSMA sample. It is presented in Fig. 5. A magnetic circuit including a coil and a ferromagnetic core permits to create a magnetic field inside an air-gap where an MSMA sample is inserted. This sample is attached at one extremity to the fixed support and at the other extremity to a mobile load. The weight of the load permits to pre-stress the MSMA sample to obtain a motion in both directions. Gravitational and inertial effects of the load have to be taken into account. The coil is supplied by an home-made switching power amplifier (200 V - $2 \mathrm{~A}$ ). The displacement of the load is measured with a laser sensor (Keyence LK-152) and the control is performed using a DSP board (dSpace). A PC is used for the displacement signal acquisition and to control the complete system.

A picture of this actuator is presented in Fig. 6. As it can be seen in the experimental system, a fulcrum and a arm lever are used to amplify the inertial effects. The working of the complete system is equivalent to the system depicted in Fig. 5 if we take into account two different masses $m_{g}$ and $m_{i}$ for the gravity and the inertial effects. Large white arrows represent all forces exerted on the bar.

\section{Energies expressions}

In this section the different energy expressions for the considered system are presented. A powerfull property of an energetic description is the property of additivity. It states

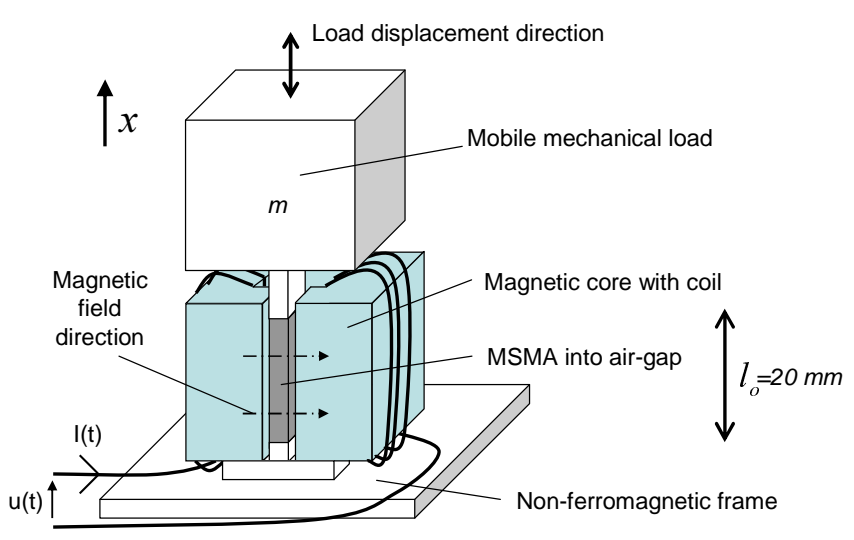

Fig. 5. General scheme of the MSMA based actuator.

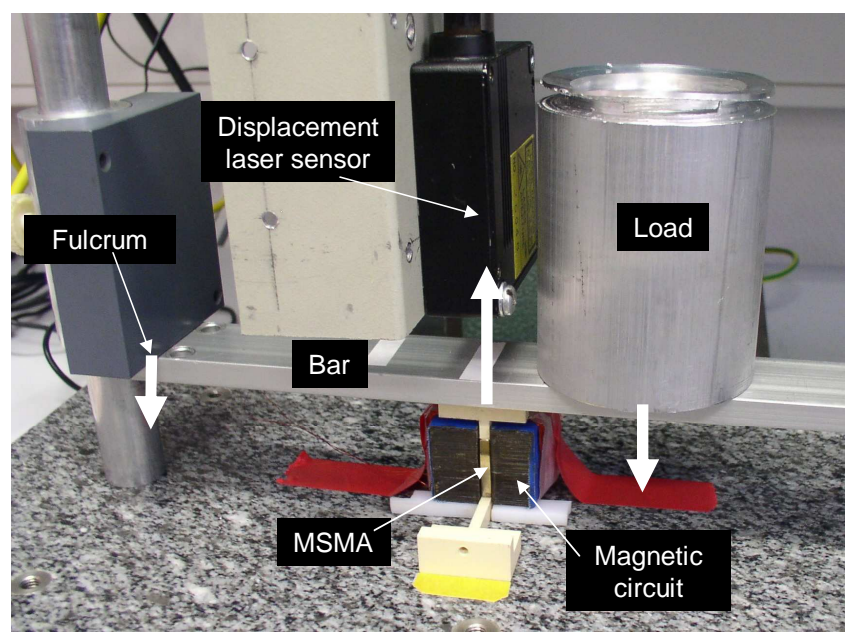

Fig. 6. Photograph of the actuator test bench.

that the total energy of one system is equal to the sum of the energy of each sub-system (magnetic circuit, MSMA, and driven load). For each sub-system, all generalized coordinates $q_{i}$, generalized momentums $p_{i}$, hamiltonian function, dissipation matrix $\mathbf{R}$, and kinematics constraints matrix $\mathbf{A}$ will be detailed. The different energies are sketched in Fig. 7.

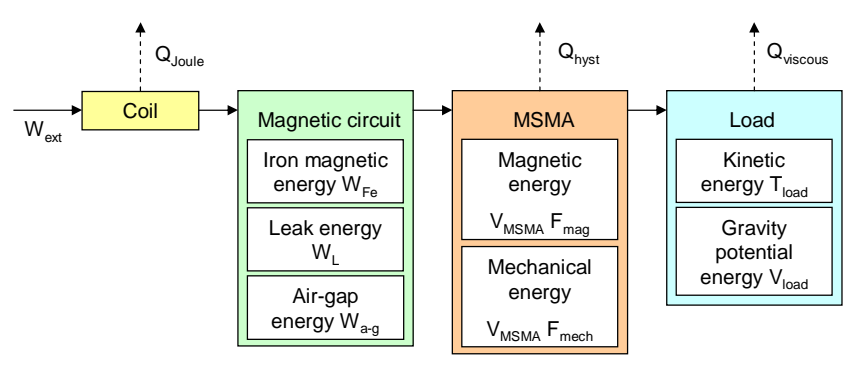

Fig. 7. Different energies of the full system.

\subsection{Magnetic circuit modelling}

In order to model the core and the magnetic circuit, we use an electrical network including resistances and inductances (see Fig. 8). This lumped-parameters model per- 
mits to take into account the magnetic leakage in the surrounding air and the ferromagnetic saturation without any time-consuming numerical computation such as the finite elements method.

\subsubsection{Definition of magnetic circuit coordinates}

A lagrangian electrostatic convention (see [16]) is chosen because it permits to consider the voltage $u(t)$ applied to the coil as an external generalized force $f_{\text {ext }}(t)$. Inductances $L_{F e}, L_{l}, L_{a}$ and $L$ are respectively associated with the magnetic flux accross the Fe-Si core, the leakage magnetic flux in the surrounding air, the magnetic flux accross the airgap, and lastly the magnetic flux accross the MSMA. It must be noticed that both inductances $L_{F e}$ and $L$ are not constant parameters but they take into account the nonlinear magnetic behaviours of these two materials. The corresponding coordinates are reported in Table 1.

\begin{tabular}{|c|c|c|c|c|}
\hline & $\mathrm{i}$ & $q_{i}$ & $\dot{q}_{i}$ & $p_{i}$ \\
\hline \hline Coil & 1 & charge $q_{c}$ & $I$ & $\phi$ \\
\hline Fe-Si core & 2 & $D_{F e}$ & $H_{F e} \cdot l_{F e}$ & $B_{F e} \cdot S_{F e}$ \\
\hline Air leakage & 3 & $D_{L}$ & $H_{L} \cdot l_{L}$ & $B_{L} \cdot S_{L}$ \\
\hline Air-gap & 4 & $D_{a-g}$ & $H_{a-g} \cdot l_{a-g}$ & $B_{a-g} \cdot S_{a-g}$ \\
\hline MSMA & 5 & $D$ & $H \cdot l$ & $B \cdot S$ \\
\hline
\end{tabular}

Table 1

Generalized coordinates, velocities and momentums used in the modelling of the magnetic circuit of the Fig. 8.

For the coil's quantities, a global form is used: the electric charge $q_{c}$ is the generalized coordinate $q_{1}$, the current $I$ is the generalized velocity $\dot{q}_{1}$, the magnetic flux $\phi$ is the generalized momentum $p_{1}$. For the other magnetic quantities, a local form is used: the magnetic excitation field $D_{i}$ corresponds to the generalized coordinate, the curvilinear integral $H_{i} \cdot l_{i}$ along the path $l_{i}$ corresponds to the generalized velocity, the flux $B_{i} \cdot S_{i}$ accross the surface $S_{i}$ is the generalized momentum. $l_{F e}, l_{l}, l_{a}$ and $l$ are respectively the ferromagnetic core, leakage, airgap and MSMA mean flux path lengths. The dissipation due to eddy-currents are neglected because the laminated Fer-Si magnetic core limits them drastically.

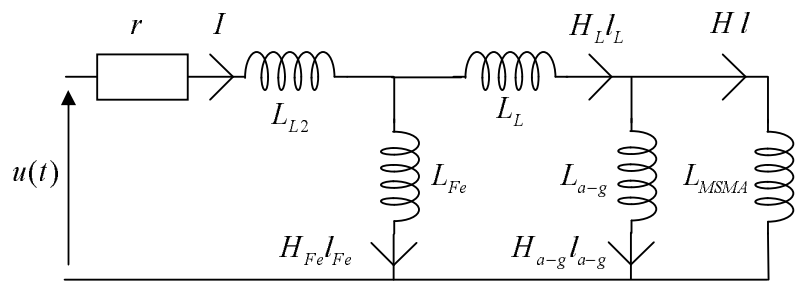

Fig. 8. Lumped-parameter model used for the modelling of electrical and magnetical quantities.

\subsubsection{Dissipation potential of the magnetic circuit}

Joule effect losses in the coil are taken into account with a dissipation potential (Rayleigh function $\mathcal{R}_{1}$ ). If $r$ is the full resistance of the coil, we have:

$$
\mathcal{R}_{1}=\frac{1}{2} r \cdot \dot{q}_{c}^{2}=\frac{1}{2} r \cdot I^{2}
$$

5.1.3. Magnetic circuit energies and hamiltonian function The magnetic energy depends on the magnetic fields $B_{i}$ (generalized momentum $p_{i}$ ) in the volume $V$ :

$$
\begin{aligned}
\mathcal{H} & =\mathcal{W}_{\text {mag }} \\
& =\int_{V} \int_{o}^{B_{i}} H_{i}(b) d b \cdot d V \\
& =\mathcal{W}_{L 2}+\mathcal{W}_{F e}+\mathcal{W}_{L}+\mathcal{W}_{a-g} \\
\mathcal{W}_{L 2}(\phi) & =\frac{\phi^{2}}{2 L_{L 2}} \\
\mathcal{W}_{F e}\left(B_{F e}\right) & =V_{F e} \cdot \int_{o}^{B_{F e}} H_{F e}(b) d b \\
\mathcal{W}_{L}\left(B_{L}\right) & =V_{L} \cdot \frac{1}{2 \mu_{o}} B_{L}^{2} \\
\mathcal{W}_{a-g}\left(B_{a-g}\right) & =V_{a-g} \cdot \frac{1}{2 \mu_{o}} B_{a-g}^{2}
\end{aligned}
$$

The magnetic energy $\mathcal{W}_{F e}$ stored in the Fe-Si core takes into account the nonlinear saturated magnetic behaviour of the Fe-Si material with an arctan shape function. The value of $\mathcal{W}_{L 2}$ is extremely smaller than these of the three other terms.

\subsubsection{External force of the magnetic circuit}

The generalized external force applied to this sub-system is the voltage applied to the coil:

$$
f_{\text {ext }}=u(t)
$$

\subsubsection{Kinematic constraints of the magnetic circuit}

Ampère's law (Kirchhoff's current law in Fig. 8) gives some algebraic relations between coordinates resulting in the definition of two kinematic constraints:

$$
\begin{aligned}
& N \cdot I=H_{F e} \cdot l_{F e}+H_{L} \cdot l_{L} \\
& \stackrel{\int d t}{\Longrightarrow} c_{1}(\mathbf{q})=D_{F e}+D_{l}-N \cdot q_{c}=0 \\
& H_{L} \cdot l_{L}=H_{a-g} \cdot l_{a-g}+H \cdot l \\
& \stackrel{\int d t}{\Longrightarrow} c_{2}(\mathbf{q})=D_{L}-D_{a-g}-D=0
\end{aligned}
$$

\subsection{MSMA modelling}

\subsubsection{Definition of MSMA coordinates}

Two types of generalized coordinates are considered in the MSMA modelling. The first ones, the temperature $T$, the strain $\varepsilon$ and the magnetic field $H$ are classical thermodynamic variables. They are associated with three thermodynamic forces, the entropy $s$, the mechanical stress $\sigma$ and the magnetization of the MSMA $M$. The coordinate $T$ is not used afterwards because of the isothermic working of the actuator and the constant value of $\mathcal{F}_{\text {therm }}$. The second type of generalized coordinates appears only in the frame of the thermodynamics of irreversible processes with internal 
variables. An internal variable is a generalized coordinate characterizing an internal working of the material not directly linked to any external forces. This variable permits to take into account the memory effect of the material. For the MSMA, the volume fraction $z$ is an internal variable. All the relevant generalized coordinates used for the modelling of the MSMA are reported in Table 2.

\begin{tabular}{|c|c|c|c|}
\hline i & $q_{i}$ & $\dot{q}_{i}$ & $p_{i}$ \\
\hline MSMA 5 & Field $D$ & $H \cdot l$ & $B \cdot S$ \\
\hline MSMA 6 & Fraction $z$ & $\dot{z}$ & $p_{z}$ \\
\hline MSMA 7 & Strain $\varepsilon$ & $\dot{\varepsilon}$ & $p_{\varepsilon}$ \\
\hline
\end{tabular}

Table 2

Generalized coordinates, velocities and momentums used in the modelling of the MSMA.

\subsubsection{MSMA energies and hamiltonian function}

The Hamiltonian function of the MSMA sample corresponds to its total energy. Because of the size and weight of the MSMA sample compared to the size and weight of the load, the influence of the potential and the kinetic energies of MSMA is quite low in the complete device energy and therefore these two terms can be neglected. The hamiltonian function of the MSMA sample can be expressed as:

$$
\mathcal{H}_{\text {MSMA }}=V_{\text {MSMA }} \cdot\left(\mathcal{F}_{\text {mech }}+\mathcal{F}_{\text {mag }}\right)+\frac{p_{z}^{2}}{2 m_{z}}+\frac{p_{\varepsilon}^{2}}{2 m_{\varepsilon}}
$$

$m_{z}$ and $m_{\varepsilon}$ are inertial parameters corresponding respectively to the $z$ and $\varepsilon$ variables. We consider them in the general case, but they will be neglected in the computation because (i) the mass of the MSMA material is very lower than the mass of the load and (ii) the process of martensite reorientation is considered as nearly instantaneous compared to the other time constants (electrical and mechanical). In the hamiltonian formalism, it is equivalent to say that $p_{\varepsilon}$ and $p_{z}$ are nearly equal to 0 . The Helmholtz free energies $\mathcal{F}_{\text {mech }}$ and $\mathcal{F}_{\text {mag }}$ are expressed in the section 2.2.2.

\subsubsection{Dissipation potential of the MSMA}

The internal variable $z$ was introduced to model the dissipative hysteretical behaviour of the material. In order to satisfy the second thermodynamic law, $z$ is used to define the Clausius-Duhem inequality:

$$
d \mathcal{D}=\pi^{f *}(z, \dot{z}) \cdot d z \geq 0
$$

with $\pi^{f *}(z, \dot{z})$ the thermodynamic force associated with $z$. A simplified expression is chosen corresponding to a static hysteresis without inner loops:

$$
\pi^{f *}(z, \dot{z})=\lambda_{C}\left[z+\frac{\operatorname{sign}(\dot{z})}{2}-\frac{1}{2}\right]+\pi^{c r} \cdot \operatorname{sign}(\dot{z})
$$

$\lambda_{C}$ corresponds to a constant kinetic parameter. $\pi^{c r}$ is an internal friction parameter. The dissipation power $\mathcal{P}_{\text {hyst }}$ is then deduced:

$$
\begin{aligned}
& \mathcal{P}_{\text {hyst }}=V_{M S M A} \cdot \dot{\mathcal{D}}=V_{M S M A} \cdot \pi^{f *}(z, \dot{z}) \cdot \dot{z} \\
& =V_{M S M A} \cdot\left(\lambda_{C} \cdot \dot{z} \cdot\left[z+\frac{\operatorname{sign}(\dot{z})}{2}-\frac{1}{2}\right]+\dot{z} \cdot \pi^{c r} \cdot \operatorname{sign}(\dot{z})\right)
\end{aligned}
$$

This power can be incorporated in the dissipation matrix $\mathbf{R}$ by adding the following term as reported in [5]:

$$
\left(\frac{\partial \mathcal{P}_{\text {hyst }}}{\partial \dot{z}}\right) / \dot{z}
$$

\subsection{Load modelling}

The driven load energy includes a kinetic energy $\left(\mathcal{T}_{\text {load }}=\right.$ $\frac{1}{2 m} p_{x}^{2}$ with $m$ the mass of the load) and a gravity potential energy $\left(\mathcal{V}_{\text {load }}=m g x\right.$ with $g$ the gravity constant $)$. The viscous friction of the load in the surrrounding air is modelled using a dissipation potential (Rayleigh function $\mathcal{R}_{2}=\frac{f}{2} \dot{x}^{2}$ with $f$ the viscous friction coefficient). The only relevant generalized coordinate for the load is its position $x$ as reported in Table 3 .

$$
\begin{array}{|c|c|c|c|c|}
\hline & \mathrm{i} & q_{i} & \dot{q}_{i} & p_{i} \\
\hline \hline \text { Load } & 8 & \text { displacement } x & \dot{x} & p_{x} \\
\hline
\end{array}
$$

Table 3

Generalized coordinate, velocity and momentum used in the modelling of the load.

Moreover the load attached to the MSMA sample gives an algebraic relation between the strain $\varepsilon$ and the displacement $x$. This leads to an other kinematic constraint:

$$
l_{0} \cdot \varepsilon=x \Rightarrow c_{3}(\mathbf{q})=x-l_{0} \cdot \varepsilon=0
$$

\section{Hamilton equations}

By adding all the previous sub-system energies, the Hamiltonian function of the full system can be written as:

$$
\begin{aligned}
& \mathcal{H}=\frac{\phi^{2}}{2 L_{L 2}}+V_{F e} \int_{o}^{B_{F e}} H_{F e}(b) d b \\
& +V_{L} \frac{1}{2 \mu_{o}} B_{L}^{2}+V_{a-g} \frac{1}{2 \mu_{o}} B_{a-g}^{2}+ \\
& V_{M S M A} \cdot\left(\frac{E}{2}(\varepsilon-\gamma z)^{2}+K_{12} \cdot z \cdot(1-z)+\int_{o}^{B} H(b) d b\right) \\
& +\frac{p_{z}^{2}}{2 m_{z}}+\frac{p_{\varepsilon}^{2}}{2 m_{\varepsilon}}+\frac{1}{2 m} p_{x}^{2}+m g x
\end{aligned}
$$

By using the port-hamiltonian representation, we have to define different vectors such as the generalized coordinates $\mathbf{q}$, momentums $\mathbf{p}$ and lagrange multipliers vector $\lambda$ :

$$
\begin{aligned}
& \mathbf{q}^{T}=\left[q_{c}, D_{F e}, D_{L}, D_{a-g}, D, z, \varepsilon, x\right] \\
& \mathbf{p}^{T}=\left[\phi, B_{F e} S_{F e}, B_{L} S_{L}, B_{a-g} S_{a-g}, B S, p_{z}, p_{\varepsilon}, p_{x}\right] \\
& \lambda^{T}=\left(\begin{array}{lll}
\lambda_{1} & \lambda_{2} & \lambda_{3}
\end{array}\right)
\end{aligned}
$$

We also have to define different matrix such as the dissipation matrix $\mathbf{R}$, the kinematic constraints matrix $\mathbf{A}$ and the external input matrix $\mathbf{B}$ : 


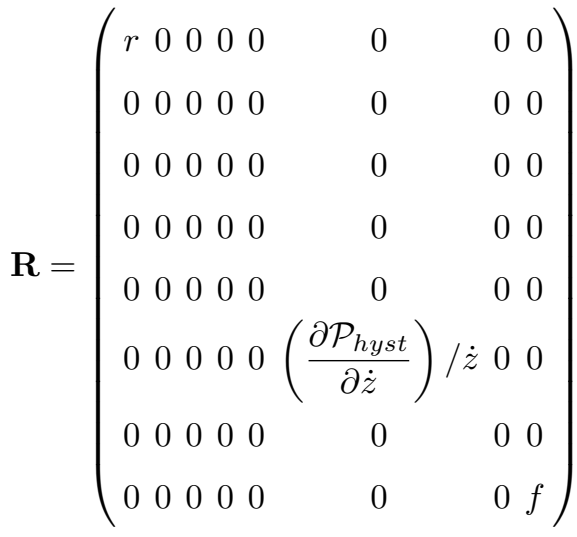

$$
\begin{aligned}
& \mathbf{A}^{T}=\left(\begin{array}{cccccccc}
-N & 1 & 1 & 0 & 0 & 0 & 0 & 0 \\
0 & 0 & 1 & -1 & -1 & 0 & 0 & 0 \\
0 & 0 & 0 & 0 & 0 & 0 & -l_{o} & 1
\end{array}\right) \\
& \mathbf{B}^{T}=\left(\begin{array}{llllllll}
1 & 0 & 0 & 0 & 0 & 0 & 0 & 0
\end{array}\right)
\end{aligned}
$$

The expression of the hamiltonian function permits to obtain 16 Hamilton equations. Among these 16 equations, 8 are associated with the time rate of coordinates and 8 with the time rate of momentums. The 8 equations associated with the time rate of coordinates gives 8 definitions of variables:

- the first Hamilton equation is the definition of the inductance $L_{L 2}$ :

$$
L_{L 2} \dot{q}_{c}=\phi
$$

- the four following Hamilton equations are the definition of the magnetic fields $H_{i}$ for $i \in\{F e, L, a-g, \varnothing\}$ :

$$
\dot{D}_{i}=l_{i} H_{i}
$$

- the three following Hamilton equations are the definition of the relations between the momentum $p_{i}$ and the velocities $\dot{q}_{i}$ for $q_{i} \in\{z, \varepsilon, x\}$ :

$$
p_{q_{i}}=m_{q_{i}} \dot{q}_{i}
$$

The 8 equations associated with the time rate of momentums give 8 relations that can be rewritten to obtain 4 physical equations (one voltage Kirchoff's law, two magnetic flux conservations and one Newton's law), one constitutive equation for the MSMA and finally the value of the three Lagrange multipliers (as denoted before, due to some simplifications, $p_{z}, p_{\varepsilon}$ and $\phi$ can be considered as nearly equal to 0$)$ :

- the dynamic electrical equation (Voltage Kirchoff's Law):

$$
u=r I+N \dot{B}_{F e} S_{F e}
$$

- two equations for the conservation of magnetic fluxes in the magnetic circuit (see Fig. 8):

$$
\left\{\begin{array}{l}
\dot{B}_{L} S_{L}=\dot{B}_{F e} S_{F e}-\dot{B}_{a-g} S_{a-g} \\
\dot{B} S=\dot{B}_{a-g} S_{a-g}
\end{array}\right.
$$

- the dynamic equation of the load (Newton's law):

$$
m \ddot{x}=-m g-f \dot{x}-S_{o} \sigma,
$$

- the quasi-static behaviour of the MSM material (the constitutive equation):

$$
\pi^{f *}(z, \dot{z})=-\gamma z+K_{12}(1-2 z)+\frac{\partial \int_{o}^{B} H(b) d b}{\partial z} .
$$

- the values of the three Lagrange multipliers:

$$
\left\{\begin{array}{l}
\lambda_{1}=\dot{B}_{F e} S_{F e} \\
\lambda_{2}=-\dot{B}_{a-g} S_{a-g} \\
\lambda_{3}=-S_{o} \sigma
\end{array}\right.
$$

\section{Parameters identification}

In order to determine some parameters of the MSMA material and some of the complete system, experimental measurements followed by an identification procedure have been performed.

\subsection{Magnetic circuit characteristics}

The magnetic circuit parameters were identified by two experiments. The first one is the measurement of the airgap magnetic field versus coil current without any MSMA sample in the airgap. These measurements are reported in Fig. 9 compared with the simulations resulting from the identification procedure.

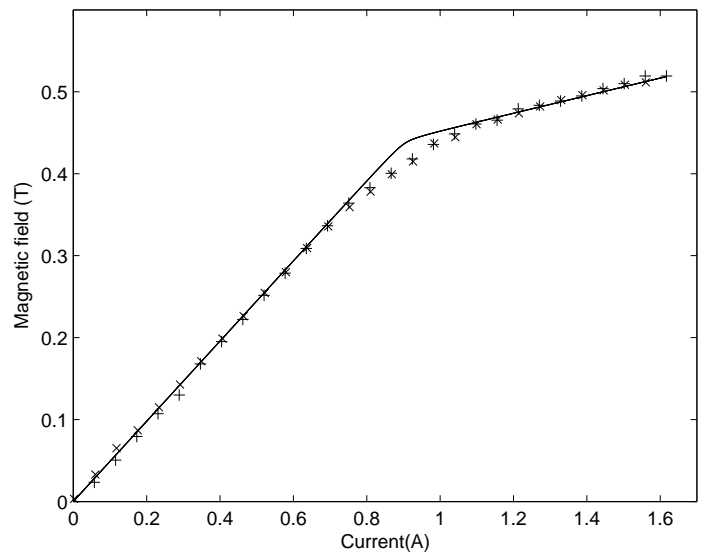

Fig. 9. First experiment for the magnetic circuit identification procedure (without MSMA): Magnetic field versus coil current (experimental measurements: + when the current is increasing and $\times$ when the current is decreasing, simulation using identified parameters: solid line).

The second experiment is the measurement of the current dynamical response versus time for a $100 \mathrm{~V}$ voltage step. This measurement is reported in Fig. 10 compared with the simulations resulting from the identification procedure.

\subsection{MSMA quasi-static characteristics}

In order to find the $\chi_{a}$ and $\chi_{t}$ parameters, the dynamical response of the magnetic circuit including the MSMA sample has been measured. A $100 \mathrm{~V}$ voltage step is applied to 


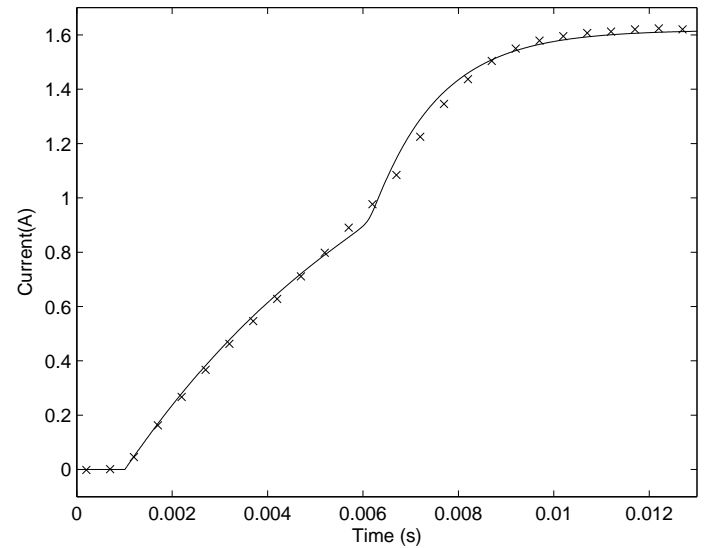

Fig. 10. Second experiment for the magnetic circuit identification procedure (without MSMA): coil current versus time for a $100 \mathrm{~V}$ voltage step (experimental measurements: cross $\times$, simulation using identified parameters: solid line).

the coil and the current is measured. In a first experiment, the MSMA was blocked to keep the martensite volume fraction $z=0$. In a second experiment, the MSMA was free of stress and initialized to $z=1$. Fig. 11 shows experimental results compared with simulations using the identified parameters. These results show that the response changes according to the $z$ value. This property can be exploited in a self-sensing application. Indeed, by measuring the current change, the volume fraction $z$ and the displacement of the load can be estimated. An example of such an application as sensor has already been studied in [17]. It uses the change of the inductance value at low magnetic field as an information for a position sensor application.

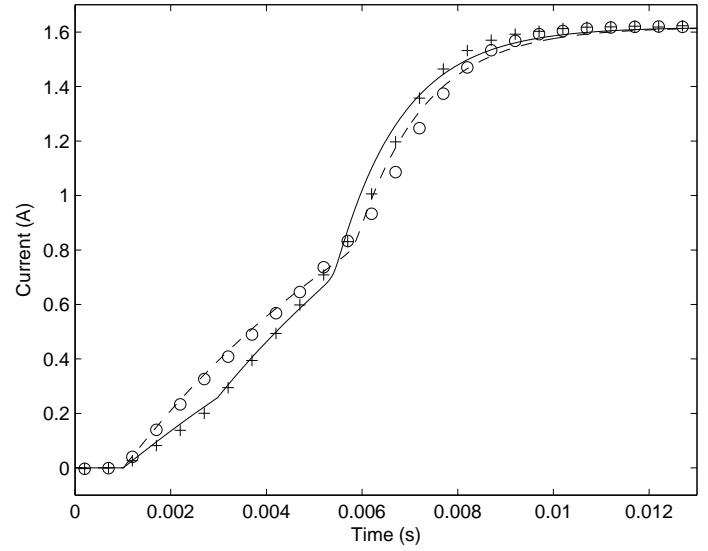

Fig. 11. Self-sensing feasibility: current versus time for a $100 \mathrm{~V}$ voltage step for $z=1$ (modelling: solid line, experimental results: cross points) and for $z=0$ (simulation: dashed line, experimental results: circles).

A compression test was performed in order to identify all MSMA mechanical parameters. Fig. 12 compares experimental and simulation results for the mechanical stress versus strain with and without a magnetic field. For a mechanical cyclic charging without a magnetic field, the mechanical behaviour is not closed (the ending point of the curve is not the starting point). This is due to the very large hysteresis of the MSMA material. But for a mechanical cyclic charging with a sufficient magnetic field $(600 \mathrm{kA} / \mathrm{m})$, the curve is closed (the ending and the starting points coincide).
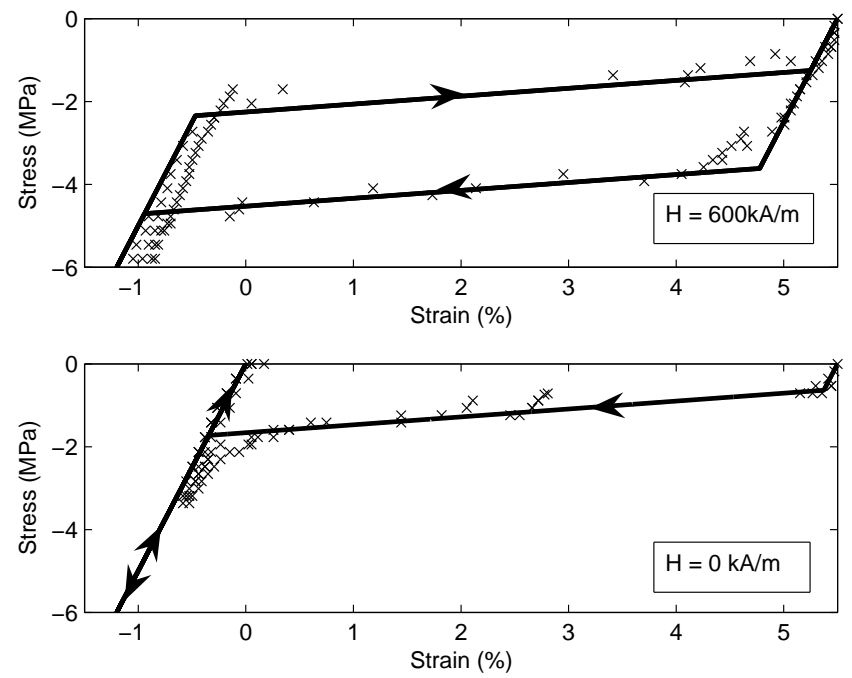

Fig. 12. Mechanical stress versus strain with and without a magnetic field for a cyclic charging (simulation: solid line, experimental results: cross points)

\subsection{Load characteristics}

The parameter $f$ was identified thanks to a dynamical response measurement reported in Fig. 14.

Table 4 summarizes all the parameters values directly measured or identified and used afterwards.

\section{Experimental validation of the model}

Numerical computations were conducted with the Matlab Simulink ${ }^{\circledR}$ software. Our simulation uses a finite differences method to compute the dynamical response of the complete device. Some S-functions programmed in C language were developed to increase the computing speed. The solver uses a classical Euler integration scheme with a sampling time of $10 \mu \mathrm{s}$.

In a first time, a sufficiently slow voltage ramp was applied as an input to verify the quasi-static model behaviour. Fig. 13 presents the voltage, current and displacement of the actuator versus time for the experimental and the simulation values. The maximum reachable displacement is about 550 micrometres after a current ramp satured at 1 A.

In a second time, a voltage step is applied to extract the dynamical behaviour of this system. Fig. 14 reports also the voltage, current and displacement versus time (this graph uses a smaller time range). The maximum reachable displacement is now about 750 micrometres for a $1 \mathrm{~A}$ current step.

One have to note that the dynamical effect combined with the nonlinear MSMA behaviour permits to obtain a 


\begin{tabular}{|c|c|}
\hline Measured & $\begin{array}{c}l_{a-g}=0.65 \mathrm{~mm} \\
l_{M S M A}=3.2 \mathrm{~mm} \\
m_{\text {grav }}=1.44 \mathrm{~kg} \\
m_{\text {inertia }}=2.32 \mathrm{~kg} \\
S=5 \times 20 \mathrm{~mm}^{2} \\
S_{\text {air }}=S \\
r=61.8 \Omega \\
N=1500 \mathrm{turns} \\
l_{o}=20 \mathrm{~mm}\end{array}$ \\
\hline $\begin{array}{l}\text { Identified } \\
\text { using Fig. } 9 \\
\text { and Fig. } 10\end{array}$ & $\begin{array}{c}S_{F e}=5 \times 23 \mathrm{~mm}^{2} \\
S_{L}=30 \times S \\
l_{L}=27 \mathrm{~mm} \\
l_{F e}=3 \mathrm{~mm}\end{array}$ \\
\hline $\begin{array}{l}\text { Identified } \\
\text { using Fig. } 11\end{array}$ & $\begin{array}{c}\chi_{t}=1 \\
\chi_{a}=40\end{array}$ \\
\hline $\begin{array}{l}\text { Identified } \\
\text { using Fig. } 12\end{array}$ & $\begin{array}{c}\gamma=0.055 \\
\lambda_{C}=130000 \mathrm{~J} / \mathrm{m}^{3} \\
\pi_{c r}=0 \mathrm{~J} / \mathrm{m}^{3} \\
K_{12}=35000 \mathrm{~J} / \mathrm{m}^{3} \\
\mu_{o} M s=0.65 \mathrm{~T} \\
E=5 e 8 \mathrm{~Pa}\end{array}$ \\
\hline $\begin{array}{c}\text { Identified with } \\
\text { dynamical results }\end{array}$ & $f=100$ \\
\hline Iron parameters & $\begin{array}{c}\chi_{F e}=7700 \\
\mu_{o} M s a t_{F e}=2.03\end{array}$ \\
\hline
\end{tabular}

Table 4

Summary of the device parameters directly measured or identified using some experiments.
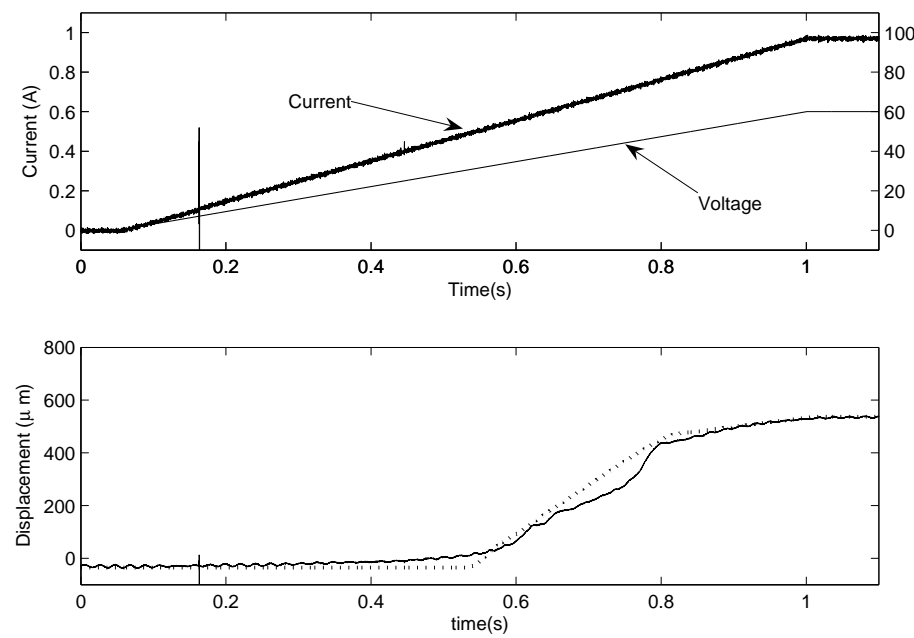

Fig. 13. Quasi-static behaviour of the system: voltage, current and displacement versus time (simulation: dotted line, experimental results: solid line).
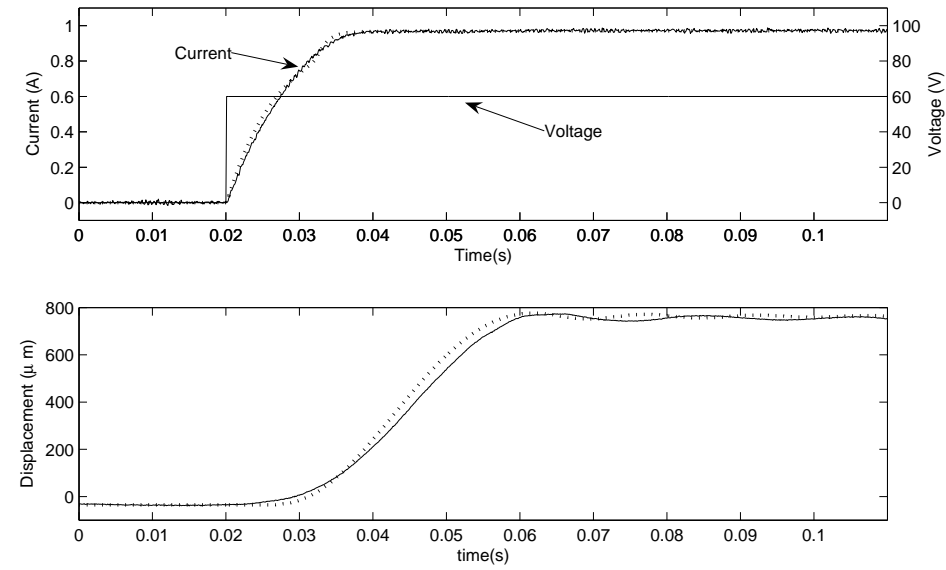

Fig. 14. Dynamic behaviour of the system: voltage, current and displacement versus time (simulation: dotted line, experimental results: solid line).

larger reachable strain in the dynamic mode than in the quasi-static mode. This is due to the decrease of the compressive stress applied to the MSMA sample when the acceleration of the load appears in dynamic mode. The simulation results appear reasonably accurate compared with the experimental measurements.

\section{Discussion on the energy distribution}

The different energies previously computed are reported in Fig. 15 first column for the quasi-static mode and in Fig. 15 second column for the dynamic mode.

\subsection{Quasi-static mode}

The first top left graph of Fig. 15 presents the electrical energy $\mathcal{W}_{\text {ext }}$ supplied to the device. As the energy exchange is $d \mathcal{W}_{\text {ext }}=u(t) \cdot d q_{c}$, the energy supply rate corresponding to the the electrical power is therefore $\dot{\mathcal{W}}_{\text {ext }}=u(t) \cdot I(t)$. The heat exchange was not measured on the bench but the ¿dissipation by Joule effect can be computed as $\mathcal{Q}_{\text {joule }}=$ 䢓 $\int_{0}^{t} \mathcal{R}_{1}\left(\dot{q}_{1}\right) d t$ and is reported in the same figure. As it can be seen, the two plots are nearly superposed and therefore the main part of the supply energy is dissipated as heat losses into the coil. This confirms that MSMA as well as classical Shape Memory Alloys are not attractive materials from the efficiency point of view.

The rest of the available energy is divided into the coil and core magnetic energies (recoverable energies, see the second top left graph of Fig. 15) and into an energy transfer to the MSMA and to the load (see the third top left graph of Fig. 15). A part of the MSMA energy is lost in the hysteretical loop of the material behavior $\mathcal{Q}_{\text {hyst }}=\int_{0}^{t} \mathcal{P}_{\text {hyst }} d t$ when the other is converted through the electromechanical energy conversion process. The result of this energy conversion is then distributed as a viscous friction process $\mathcal{Q}_{\text {viscous }}=\int_{0}^{t} \mathcal{R}_{2}(\dot{x}) d t$, a potential energy $\mathcal{V}_{\text {load }}(x)$ and a kinetic energy $\mathcal{T}_{\text {load }}\left(p_{x}\right)$. We can see in the bottom left 

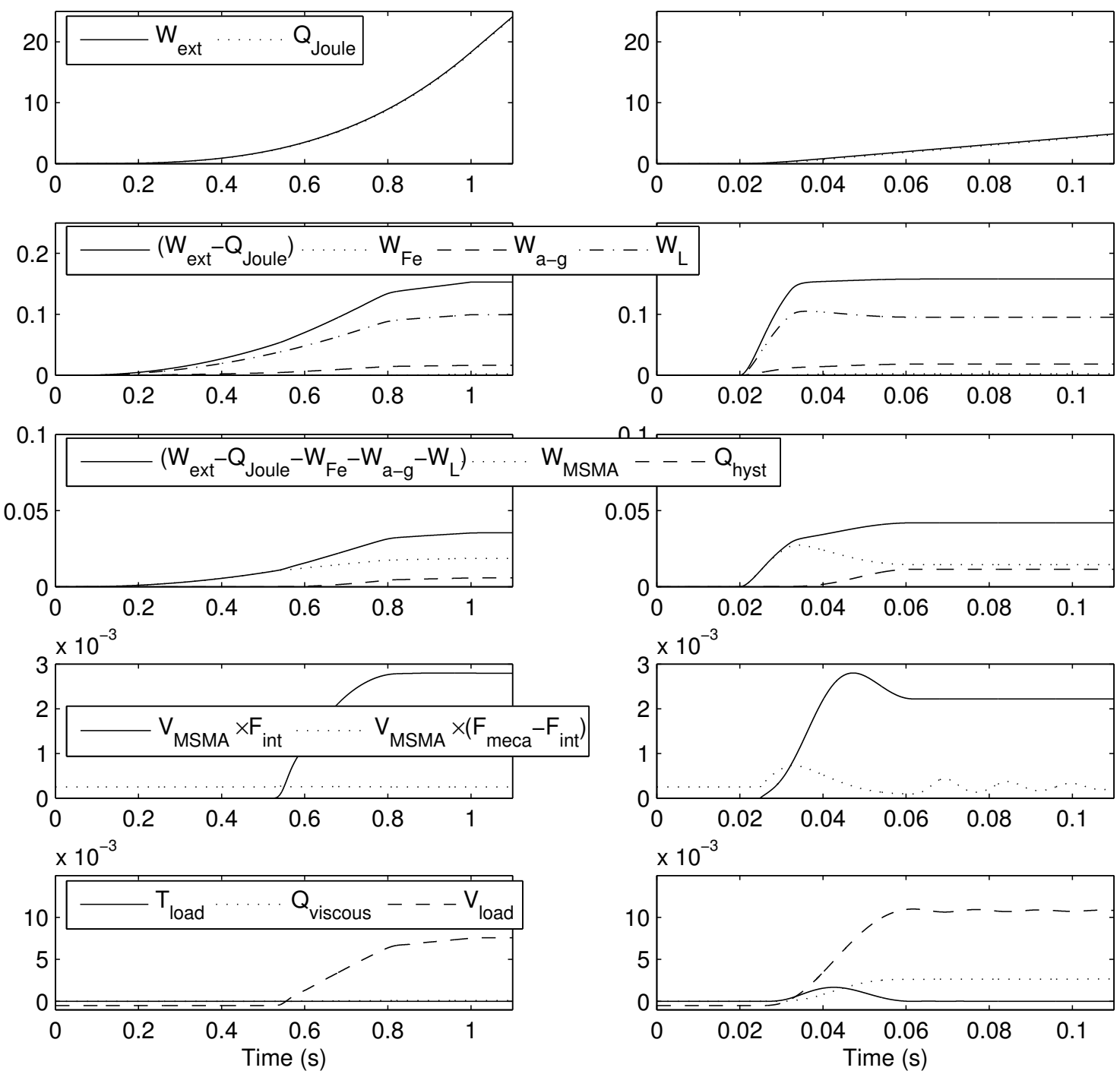

Fig. 15. Computations of the different energies (Joules) versus time : in the static mode (left column) and in the dynamic mode (right column).

graph of Fig. 15 that the practical available mechanical energies (kinetic $\mathcal{T}_{\text {load }}$ and potential $\mathcal{V}_{\text {load }}$ ) are quite small compared to the input energy. The MSMA elastic energy $\mathcal{F}_{\text {mech }}-\mathcal{F}_{\text {int }}$, the kinetic energy $\mathcal{T}_{\text {load }}$, and viscous losses $\mathcal{Q}_{\text {viscous }}$ are constant because of the quasi-static mode.

\subsection{Dynamic mode}

The first top right graph of Fig. 15 shows also clearly that, in this system, the supplied energy is mainly dissipated by heat losses into the coil. The differences between dynamic mode and quasi-static mode are now discussed. In $\mathcal{F}_{\text {mech }}$, the martensite interaction energy $\mathcal{F}_{\text {int }}=K_{12} \cdot z \cdot(1-z)$ reaches a maximum when the $z$ value is equal to 0.5 . An energy transfer between the MSMA elastic energy $\frac{E}{2} \cdot(\varepsilon-\gamma \cdot z)^{2}$ and the kinetic energy $\mathcal{T}_{\text {load }}$ exists. Actually, this elastic energy increases when some energy is supplied to the system, then decreases to a lower value than beginning because of kinetic energy $\mathcal{T}_{\text {load }}$. At this time, $\mathcal{V}_{\text {load }}$ can increase to a higher value than in quasi-static mode. The dissipation is more important for $\mathcal{Q}_{\text {hyst }}$ and $\mathcal{Q}_{\text {viscous }}$ in the dynamic case than in the quasi-static but $\mathcal{Q}_{\text {joule }}$ is lower in the dynamic case because the time range is smaller than in quasi-static mode.

\subsection{Energy efficiency}

Less than 8 to $11 \mathrm{~mJ}$ are recovered by the load for 5 to 20 Joules supplied to the actuator. These results clearly show a poor actuator efficiency. Because the main energetic losses are due to the Joule effect, an efficient actuator is an actuator which can hold a displacement value without keeping a current into the coil. This problem can be partially solved by using a Push-Pull actuator design working by voltage pulses: two MSMA samples and two magnetic circuits are used in an antagonistic way in order to obtain a multi-stable actuator. This kind of actuator was also designed and studied by the authors in [18]. 


\section{Conclusion and perspectives}

In this paper, a lagrangian formalism and its hamiltonian extension combined with the thermodynamics of irreversible processes were used to obtain the complete dynamical modelling of a mechatronic device including Magnetic Shape Memory Alloys. This model permits to simulate the complete dynamical behaviour of this system even if the MSMA behaviour is nonlinear and hysteretic. The self-sensing feasibility and an estimation of the different energies were given. All these results are experimentally validated and a satisfactory accuracy between simulations and experimental results is observed. The model can be used confidently in the near future in order to design a new controller using some energy based control techniques such as it is proposed in [3], [4], [5] and [6].

\section{References}

[1] J. L. Pons, Emerging Actuator Technologies: A Micromechatronic Approach, John Wiley and Sons, 2005.

[2] J. Y. Gauthier, C. Lexcellent, A. Hubert, J. Abadie, N. Chaillet, Modeling rearrangement process of martensite platelets in a magnetic shape memory alloy $\mathrm{Ni}_{2} \mathrm{MnGa}$ single crystal under magnetic field and (or) stress action, Journal of Intelligent Material Systems and Structures 18 (3) (2007) 289-299.

[3] R. Sepulchre, M. Janković, P. Kokotović, Constructive Nonlinear Control, Springer-Verlag, 1997.

[4] R. Ortega, A. Loria, P. J. Nicklasson, H. S. Ramirez, Passivitybased Control of Euler-Lagrange Systems, Springer-Verlag, 1998.

[5] A. V. der Schaft, $\mathrm{L}_{2}$-Gain and Passivity Techniques in Nonlinear Control, Springer-Verlag, 2000.

[6] A. Astolfi, A. J. V. der Schaft (Eds.), Lagrangian and Hamiltonian Methods for Nonlinear Control, Special Issue of European Journal of Control, Volume 10, Number 5, Hermes, 2004.

[7] K. Ullakko, J. K. Huang, C. Kantner, R. C. O'Handley, V. V. Kokorin, Large magnetic-field-induced strains in ni2mnga single crystals, in: Applied Physics Letters, Vol. 69, 1996, pp. 19661968.

[8] R. D. James, M. Wuttig, Magnetostriction of martensite, in: Philosophical Magazine A, Vol. 77, 1998, pp. 1273 - 1299.

[9] S. Leclercq, C. Lexcellent, A general macroscopic description of the thermomechanical behavior of shape memory alloys, J. Mech. Phys. Solids 44(6) (1996) 953-980.

[10] L. Hirsinger, C. Lexcellent, Modelling detwinning of martensite platelets under magnetic and (or) stress actions in ni-mn-ga alloys, Journal of Magnetism and Magnetic Materials 254-255 (2002) 275-277.

[11] C. Lanczos, The Variational Principe of Mechanics, Fourth edition, Dovers Publications, Reprint of 1970 University of Toronto Press, 1986.

[12] H. Goldstein, Classical Mechanics, second edition, Addison Wesley, 1980.

[13] S. H. Crandall, D. C. K. andEdward F. Kurtz Jr., D. C. Pridmore-Brown, Dynamics of Mechanical and Electromechanical Systems, Krieger Publishing, Malabar, Reprint of 1968 McGraw Hill, 1982.

[14] A. Preumont, Mechatronics : Dynamics of Electromechanical and Piezoelectric Systems, Springer Verlag, 2006.

[15] B. Nogarede, Électrodynamique Applique, Dunod, 2005.

[16] P. Hammond, Energy Methods in Electromagnetism, Clarendon Press - Oxford University Press, 1981.
[17] I. Suorsa, E. Pagounis, K. Ullakko, Position dependent inductance based on magnetic shape memory materials, Sensors and Actuators A 121 (2005) 136-141.

[18] J. Y. Gauthier, A. Hubert, J. Abadie, C. Lexcellent, N. Chaillet, Multistable actuator based on magnetic shape memory alloy, in: ACTUATOR 2006, 10th International Conference on New Actuators, Bremen, Germany, 2006, pp. 787-790. 


\section{List of Figures}

1 MSMA behaviour: (a) austenite phase and the three martensite variants, (b) martensitic reorientation: effects of mechanical stress, magnetic field and temperature.

2 Representative elementary volume, arrows represent local domains magnetizations [10]. The thermodynamic force $\pi^{f *}$ according to the internal variable $z$ for an hysteretical behaviour. $\quad 4$ Variation of the action in the Hamilton principle.

General scheme of the MSMA based actuator.

Photograph of the actuator test bench.

Different energies of the full system.

Lumped-parameter model used for the modelling of electrical and magnetical quantities.

First experiment for the magnetic circuit identification procedure (without MSMA): Magnetic field versus coil current (experimental measurements: + when the current is increasing and $\times$ when the current is decreasing, simulation using identified parameters: solid line).

10 Second experiment for the magnetic circuit identification procedure (without MSMA): coil current versus time for a $100 \mathrm{~V}$ voltage step (experimental measurements: cross $\times$, simulation using identified parameters: solid line).

11 Self-sensing feasibility: current versus time for a $100 \mathrm{~V}$ voltage step for $z=1$ (modelling: solid line, experimental results: cross points) and for $z=0$ (simulation: dashed line, experimental results: circles).

12 Mechanical stress versus strain with and without a magnetic field for a cyclic charging (simulation: solid line, experimental results: cross points)

13 Quasi-static behaviour of the system: voltage, current and displacement versus time (simulation: dotted line, experimental results: solid line).

14 Dynamic behaviour of the system: voltage, current and displacement versus time (simulation: dotted line, experimental results: solid line).

15 Computations of the different energies (Joules) versus time : in the static mode (left column) and in the dynamic mode (right column).

\section{List of Tables}

1 Generalized coordinates, velocities and momentums used in the modelling of the magnetic circuit of the Fig. 8.

2 Generalized coordinates, velocities and momentums used in the modelling of the MSMA.

4 Summary of the device parameters directly measured or identified using some experiments. 